\title{
Magnitude of Intestinal Parasitosis and Associated Factors in Rural School Children, Northwest Ethiopia
}

\author{
Megbaru Alemu ${ }^{*}$, Abay Anley², Kiros Tedla ${ }^{3}$
}

\author{
OPEN ACCESS \\ Citation: Megbaru Alemu, Abay Anley, \\ Kiros Tedla. Magnitude of Intestinal \\ Parasitosis and Associated Factors in \\ Rural School Children, Northwest \\ Ethiopia. Ethiop J Health Sci. 2018;29 \\ (1):923. \\ doi:http://dx.doi.org/10.4314/ejhs.v29i1.14 \\ Received: February 06, 2018 \\ Accepted: April 02, 2018 \\ Published: January 1, 2019 \\ Copyright: (C) 2018 Megbaru Alemu, et \\ al. This is an open access article \\ distributed under the terms of the Creative \\ Commons Attribution License, which \\ permits unrestricted use, distribution, and \\ reproduction in any medium, provided the \\ original author and source are credited. \\ Funding: Bona District Health Bureau \\ and Abem private clinic. \\ Competing Interests: The authors declare \\ that this manuscript was approved by all \\ authors in its form and that no competing \\ interest exists. \\ Affiliation and Correspondence: \\ ${ }^{1}$ Department of Medical Laboratory \\ Science, Bahir Dar University, \\ Ethiopia \\ ${ }^{2}$ GobgobHealth Center, South Gonder, \\ Ethiopia \\ ${ }^{3}$ Department of Medical Parasitology \\ and Vector Biology, Mekelle \\ University, Ethiopia \\ *Email: mgbeyney@gmail.com
}

\author{
ABSTRACT
}

BACKGROUND: Intestinal parasitoses are among the most commonly encountered infections among school children in poor regions of the world. Up to 600 million school children are living in areas where there is high transmission of parasitic worms. Intestinal parasitic infection has been found to have a great effect on nutritional and cognitive status, school absenteeism and dropouts among school age children. This study aimed at determining the prevalence of intestinal parasites infections and associated factors among children in a rural primary school, Northwest Ethiopia.

METHODS: A cross-sectional study was conducted in May 2016 among Gob Gob Primary School children. The study participants were provided with labeled stool cups to give stool specimen. The stool samples were processed via direct wet mount and formal-ether concentration techniques. A structured questionnaire was used to collect demographic data and data on factors associated with intestinal parasitic infection through face-to-face interview.

RESULTS: Out of the 273 school children, 84(30.8\%) were infected with at least one parasite species. Higher proportion of intestinal parasitic infection was recorded for boys (38.9\%), the age group 610 years (38.9\%), children with untrimmed finger nails (36.4\%) and among those whose drinking water was from a stream (56.1\%). The predominant species identified were A. lumburicoides 28(33.3\%), H. nana 12(14\%), E. histolytica/dispar 11(13\%), G. lamblia 9(11\%), hookworms 7(8.3\%), Taenia spp 6(7\%), E. vermicularis 6(7\%), T. trichuria 4(4.8\%) and S. stericoralis 1(1.2\%).

CONCLUSION: This study showed that intestinal parasites were prevalent among the school children in focus. Ascaris lumbricoides was the predominant species.

KEYWORDS: Prevalence, Parasite, Gob Gob, Ethiopia

\section{INTRODUCTION}

Intestinal parasitic infections are among the most common infections worldwide. It is estimated that some 3.5 billion people are affected and that 450 million are ill as a result of these infections (1). It is estimated that Ascaris lumbricoides, hookworm and Trichuris trichiura infect 1.5 billion, 1.3 billion and 1.1 billion people 
worldwide, respectively, while schistosomiasis affects over 200 million people $(1,2)$. Entamoeba histolytica and Giardia lamblia are also estimated to infect about 60 million and 200 million people worldwide, respectively (3). In children, intestinal parasitic infections, particularly soil-transmitted helminthiasis is of great health concern, because children habitually play in fecally contaminated soil where infective helminth eggs/larvae are found (4). In addition to considerable mortality and morbidity, infection with intestinal helminthes has been found to greatly affect a child's mental growth and physical fitness while also predisposing children to other infections $(1,5)$. Several factors like climatic conditions, poor sanitation, unsafe drinking water and lack of toilet facilities are the main contributors to the high prevalence of intestinal parasites in tropical and sub-tropical countries (6).

Intestinal parasitism has been widespread in Ethiopia (5,6,7,9,12-14). Parasitic helminthic infections are the second most predominant causes of outpatient morbidity in the country. Several studies indicated that the prevalences of parasitic infections were high in the lower altitudes including North western parts of Ethiopia (5). Many reports illustrated that $A$. lumbricoides is the most prevalent intestinal parasite in different communities usually occurring together with T.trichuria infections (7). Shistosomiasis is common in Northren region compared to south and southwestern parts of the country. The prevalence of Taeniasis alone ranges from $1-48 \%$, and the infection rate with Hymenolopis nana is 3-61\%. Amoebiasis and Giardiasis are also common causes of intestinal protozoan infections throughout the nation. The prevalence of amoebiasis ranges from $0-4 \%$ and that of Giardiasis is $3-23 \%(5,7,8,10)$.

The probability of developing diseases caused by intestinal parasitic infections to the health and welfare of the individuals and the communities depends on parasitic species and numerous socio-economic factors among others. There are several risk factors for intestinal parasitosis, some of which are hand washing habits before meal, frequency of footwear, source of drinking water, parents' occupation, latrine availability and usage, and residence (rural/urban). Thus, the distribution of each parasite species must be evaluated at regional or local levels according to their prevalence $(1,5,7)$. Therefore, this study aimed at determining prevalence and associated factors of intestinal parasitosis among Gob Gob Primary School children, Northwest Ethiopia.

\section{METHODS AND MATERIALS}

Study design and area: This cross-sectional study was conducted among Gob Gob Primary School children in 2016. Gob Gob is a rural kebele found in Lay Gayint Woreda, South Gondar Zone, Amhara National Regional State, about 724 km from Addis Ababa. There were a total of 5,093 (2057 males and 3036 females) inhabitants in the town. There was only one primary school with a total of 273 pupils.

Sample size and sampling technique: The sample size was determined using single population proportion estimate $\left(\mathrm{z}^{2} \mathrm{p}(1-\mathrm{p}) / \mathrm{d}^{2}\right)$ considering $95 \%$ confidence level, 0.5 proportion of intestinal parasitosis (IP) and marginal error of (5\%). Thus, a sample size of 384 was obtained. However, the expected total sample size in the selected target areas is less than the calculated sample, and hence we included all the school children.

Data collection: Data was collected using structured questionnaire via face-to-face interview and observations for foot wearing and finger nail status. The interview included information on demographic characteristic of school children and factors associated with intestinal parasitosis.

Stool examination: The school children were supplied with labeled stool containers with tight covers bearing serial numbers of the subjects. The entire stool samples were received at the spot at an organized central place and processed via direct wet mount and formol ether concentration (FEC) techniques. In the FEC procedure, approximately $0.5 \mathrm{~g}$ of faeces was mixed with $10 \mathrm{ml}$ of normal saline and the mixed stool was strained via gauze in to a funnel. The strained contents were collected into a centrifuge tube. About $2.5 \mathrm{ml}$ of $10 \%$ formaldehyde and $1 \mathrm{ml}$ of ether were then added and centrifuged at $1000 \mathrm{~g}$ for 3 minutes. The supernatant was removed and a drop of the sediment was covered with cover glass for

DOI: http://dx.doi.org/10.4314/ejhs.v29i1.14 
microscopic investigation (27). In the direct wet mount preparation, on the other hand, fresh stool samples (approximately $2 \mathrm{mg}$ ) were put on a slide with wooden applicator, emulsified with a drop of physiological saline $(0.85 \%)$, and covered with cover slide and examined at $10 \mathrm{x}$ and $40 \mathrm{x}$ microscopic objectives (27).

Quality control: The questionnaire was pre-tested on a small number of children in another school in Lay Gaint woreda. The collected data were checked daily for consistency and accuracy. Standardized procedures were strictly followed during collection and processing of faecal specimens.

Data analysis: Data were entered into and analyzed using SPSS statistical software package version 20 (IBM Comp. released 2011.IBM SPSS statistics for windows, version 20 Armonk, NY: IBM comp.). Descriptive statistics was used to describe study participants in relation to relevant variables. Chi-square test was employed to measure association of dependent and independent variables. P-value less than 0.05 was taken as statistically significant.

Ethical consideration: This research was ethically approved by the Institutional Review Board (IRB) of Mekelle University, College of Health Sciences. Written consents and assents were collected, respectively, from parents/guardians and the participanting children. School children who were found to harbor any of the intestinal parasites were treated with anti-parasitic agents.

\section{RESULTS}

A total of 273 school children were enrolled in the survey of intestinal parasitosis. The mean age of the study participants was 11.7 years. Males and those above 15 years of age accounted for $148(54.2 \%)$ and $102(37.4 \%)$ of the study participants, respectively. The overall prevalence of IP and co-infection (double infection) was $30.8 \%$ and $2.9 \%$, respectively (Table 1 ).

Table 1: Prevalence and associated factors of intestinal parasitosis among Gob gob primary school children, Northwest Ethiopia

\begin{tabular}{|c|c|c|c|c|c|}
\hline Variable & Positive n (\%) & Negative n (\%) & Total n (\%) & P-value & $\mathrm{X}^{2}$ \\
\hline \multicolumn{6}{|l|}{ Age (Yrs) } \\
\hline $6-10$ & $37(38.9)$ & $58(61.1)$ & $95(34.8)$ & 0.001 & 13.4 \\
\hline $11-15$ & $22(28.9)$ & $54(71.1)$ & $76(27.8)$ & & \\
\hline $15+$ & $25(24.5)$ & $77(75.5)$ & $102(37.4)$ & & \\
\hline \multicolumn{6}{|l|}{ Gender } \\
\hline Male & $51(38.9)$ & $97(61.1)$ & $148(54.2)$ & 0.06 & 1.6 \\
\hline Female & $33(26.4)$ & $92(73.6)$ & $125(45.8)$ & & \\
\hline \multicolumn{6}{|l|}{ Finger nail status } \\
\hline Trimmed & $52(28.1)$ & $133(71.9)$ & $185(67.7)$ & 0.20 & 1.9 \\
\hline Untrimmed & $32(36.4)$ & $56(63.6)$ & $88(32.3)$ & & \\
\hline \multicolumn{6}{|c|}{ Drinking water source } \\
\hline Stream & $32(56.1)$ & $25(43.9)$ & $57(20.9)$ & 0.00 & 24.4 \\
\hline Well & $4(50.0)$ & $4(50)$ & $8(2.9)$ & & \\
\hline Pipe & $48(23.1)$ & $160(76.9)$ & $208(76.2)$ & & \\
\hline \multicolumn{6}{|c|}{$\begin{array}{l}\text { Hand washing before } \\
\text { meal }\end{array}$} \\
\hline Usually & $53(26.1)$ & $150(73.9)$ & $203(74.4)$ & 0.005 & 8.1 \\
\hline Occasionally & $31(44.3)$ & $39(55.7)$ & $70(25.6)$ & & \\
\hline \multicolumn{6}{|l|}{ Presence of toilet } \\
\hline Yes & $51(26.6)$ & $141(73.4)$ & $192(70.3)$ & 0.025 & 6.3 \\
\hline No & $33(40.7)$ & $45(55.6)$ & $81(29.7)$ & & \\
\hline $\begin{array}{l}\text { A total of } 9 \text { IP } \\
\text { lumbricoides }\end{array}$ & e identifie & $\begin{array}{l}\text { ly: } A . \\
, \quad E .\end{array}$ & $\begin{array}{l}\text { ca/dispar } \\
\mathrm{rms} \quad\left(2.6^{\circ}\right.\end{array}$ & $\begin{array}{r}0 \%), \quad G . \\
\text { Taenia }\end{array}$ & $\begin{array}{ll}\text { umblia } & (3.3 \%), \\
\text { eecies } & (2.2 \%),\end{array}$ \\
\hline
\end{tabular}

DOI: http://dx.doi.org/10.4314/ejhs.v29i1.14 
E.vermicularis $(2.2 \%)$, T.trichuria $(1.5 \%)$ and $S$. stercoralis $(0.4 \%)$. Higher proportions of IP infection were recorded for boys (38.9\%), the age group of $6-10$ years $(38.9 \%)$, children with untrimmed finger nails (36.4\%) and among those whose drinking water was from a stream $(56.1 \%)$. School children who washed their hands only occasionally prior to meal were also more affected by IPs than their counter parts $(44.3 \%)$. A. lumbricoides $(10.8 \%), H$. nana $(4.7 \%), E$. histolytica/dispar (4.7\%), G. lamblia (4.0\%), hookworms $(2.7 \%)$, T. trichuria $(2.0 \%)$ and Taenia spp (2.7\%), were more prevalent in males than females. Enterobius vermicularis $(2.4 \%)$ on the other hand was more prevalent among female participants (Table 2).

Table 2: Distribution of intestinal parasite species by sex among Gob gob primary school children Northwest Ethiopia

\begin{tabular}{llll}
\hline \multirow{2}{*}{ IP species } & Sex & & \\
\cline { 2 - 3 } Single infection & Male (n=148) & Female (n=125) & Total(n=273) \\
A. lumbricoides & $16(10.8 \%)$ & $12(9.6 \%)$ & $28(10.3 \%)$ \\
H. nana & $7(4.7 \%)$ & $5(4.0 \%)$ & $12(4.4 \%)$ \\
E. histolytica/dispar & $7(4.7 \%)$ & $4(3.2 \%)$ & $11(4.0 \%)$ \\
G. lamblia & $6(4.0 \%)$ & $3(2.4 \%)$ & $9(3.3 \%)$ \\
Hookworms & $4(2.7 \%)$ & $3(2.4 \%)$ & $7(2.6 \%)$ \\
Taenia spp & $4(2.7 \%)$ & $2(1.6 \%)$ & $6(2.2 \%)$ \\
E. vermicularis & $3(2.0 \%)$ & $3(2.4 \%)$ & $6(2.2 \%)$ \\
T. trichuria & $3(2.0 \%)$ & $1(0.8 \%)$ & $4(1.5 \%)$ \\
S.stercoralis & $1(0.7 \%)$ & - & $1(0.4 \%)$ \\
Double infection & & & \\
A. lumbricoides + H. nana & $2(1.4 \%)$ & $1(0.8 \%)$ & $3(1.1 \%)$ \\
E. histolytica /dispar + G. lamblia & $1(0.7 \%)$ & $1(0.8 \%)$ & $2(0.7 \%)$ \\
Taenia spp + H. nana & $1(0.7 \%)$ & - & $1(0.4 \%)$ \\
A.lumbricoides + E. vermicularis & $1(0.7 \%)$ & - & $1(0.4 \%)$ \\
T. trichuria + H. nana & $1(0.7 \%)$ & - & $1(0.4 \%)$ \\
\hline
\end{tabular}

The prevalence of each parasite species also varies among different age groups. For instance, the 6-10 age group harbored higher rate of infection by $A$. lumbricoides $(15.8 \%), \quad H$. nana $(7.4 \%), \quad E$. histolytica/dispar (7.4\%) and G. lamblia (5.3\%). Taenia spp (1.3\%) and S. stercoralis (1.3\%) infection on the other hand were relatively higher among the age group of 11-15 years, while hookworm infection (3.9 $\%$ was more prevalent among older children $(>15$ years) (Table 3).

\section{DISCUSSION}

The prevalence of the parasite at any given time and place is determined by the combined effect of sources of infection, mode of transmission, and presence of susceptible host (10). Intestinal parasitic infections persist and flourish whenever poverty, low level of environmental sanitation, insufficient health care and overcrowding are entrenched (7,11). A parasitological survey under taken in different parts of the world confirmed that intestinal parasitic infections have wide distribution with various degrees of prevalence $(5,12)$. This study attempted to assess the prevalence of IP infections in Gob Gob Primary School children in Northwest Ethiopia. The results of this study showed the occurrence of several IP species of public health importance among the school children.

The overall prevalence of IP in this study (30.8\%) was lower than results reported from Northern Gondar (79.8\%) (14), Northwest Ethiopia (62.3\%) (15) and Southern Ethiopia (85.1\%) (9). Moreover, higher prevalences than the one in this study were reported in Nigeria (52\%) (16) and Vietnam (88\%) (17). The lower result in this study might be due to periodic de-worming programs in the area. In contrast, the present finding was higher than results from different localities of Ethiopia $(18,19)$ and other parts of the world $(20,21)$. This might be attributable to differences in awareness regarding transmission and prevention of intestinal

DOI: http://dx.doi.org/10.4314/ejhs.v29i1.14 
Table 3: Distribution of intestinal parasite species by age among Gob gob primary school children Northwest Ethiopia.

\begin{tabular}{llll}
\hline & \multicolumn{2}{l}{ Age $($ Yrs $)$} & $15+(\mathrm{n}=102)$ \\
\cline { 2 - 4 } IP species & $6-10(\mathrm{n}=95)$ & $11-15(\mathrm{n}=76)$ & $5(4.9 \%)$ \\
A. lumbricoides & $15(15.8 \%)$ & $8(10.5 \%)$ & $2(1.98 \%)$ \\
H. nana & $7(7.4 \%)$ & $3(3.95 \%)$ & $1(0.98 \%)$ \\
E. histolytica/dispar & $7(7.4 \%)$ & $3(3.95 \%)$ & $1(0.98 \%)$ \\
G. lamblia & $5(5.3 \%)$ & $3(3.95 \%)$ & $4(3.9 \%)$ \\
Hookworms & $2(2.1 \%)$ & $1(1.3 \%)$ & $5(4.9 \%)$ \\
Taenia spp & - & $1(1.3 \%)$ & - \\
E. vermicularis & $4(4.2 \%)$ & $2(2.6)$ & - \\
T. trichuria & $4(4.2 \%)$ & - & - \\
S. stercoralis & - & $1(1.3 \%)$ & \\
\hline
\end{tabular}

parasites, environmental and personal hygiene, source of households' water supply and habit of walking barefooted. It could also be due to the local endemicity and geographic condition of the study area. The sensitivity of stool examination procedures might also play a role in the difference of IP prevalence.

Ascaris lumbricoides is the most cosmopolitan and the most common parasite to infect humans in Ethiopian (10). Our study also supported that $A$. lumbricoides was the predominant species with a prevalence of $10.3 \%$. It goes in agreement with a study in Southern Ethiopia (22). However, it was lower than reports from Jimma (23) and Northwest Ethiopia (13). The distribution is affected by altitude, being more common in higher and more humid than lower and dry areas. It is mostly associated with poverty, illiteracy, crowding, poor sanitation and personal hygiene $(7,13)$.

The prevalence of $E$. histolytica infection in this study $(4.0 \%)$ was in agreement with studies from Jimma (5.6\%) (23) and Saudi Arabia (4.7\%) (21). However, the current finding was lower than a report from Northwest Ethiopia (16.3\%) (13) and Kenya (74.1\%) (24), and higher than a result from Malaysia $(0.4 \%)$ (25). The relatively higher prevalence of E. histolytica/dispar infection in this study might be due to poor sanitary condition, poor access to safe drinking water supplies which was supported by the fact that a significant number of study participants $(56.1 \%)$ reported that the households' water for drinking was mainly obtained from streams.

The peak prevalence of IP was recorded for the age group of 6-10 years. It was in agreement with studies from Egypt (26). However, contradictory findings were reported from Southern Ethiopia (27). Even though the overall prevalence of IP was higher among children in the age group of 6-10 years, the peak hookworm infection was found in participants agred +15 years. Contradictory findings were reported from
Southern Ethiopia (5) and Northern Gonder (14) in which the hookworms were prevalent rather in the younger age groups. On the other hand, our finding was in line with studies conducted in Northwest Ethiopia (15) and Nigeria (16). The persistence of hookworms with age might be attributed to ignorance of foot wares among adult agrarian males. Hookworm infestation results from the penetration of the skin by filariform larvae and is seen in age groups that are old enough to walk bare-footed in such fields (16).

The distribution of E. histolytica and G. lamlia intestinal infections in this study was more prevalent among the younger age group (6-10). This might possibly be due to increased exposure of children in this age category to infective stages with increased mobility and weaning practices. The tendency of this age group to eat unwashed fruits, which may be contaminated with protozoan cysts, might have also contributed to the higher prevalence of E. histolytica in this age group.

\section{ACKNOWLEDGEMENT}

We would like to acknowledge Mekelle University for logistic supports.

\section{REFERENCES}

1. WHO Expert Committee. Prevention and control of schistosomiasis and soil-transmitted helminthiases. WHO Technical Report Series, 2002; 912:1-57.

2. Murray PR, Rosenthal KS, Kobayashi G.S, Pfalle HA. Medical Microbiology 4th ed. London, Mosby, 2002: 681-761.

3. Rao V, Sugunan A, Murhekar M, Sehgal S. Malnutrition and high childhood mortality among the Onge tribe of the Andaman and Nicobar Islands. Public Health Nutr 2006; 9:19-25.

4. Mahfouz AAR, El-Morshedy H, Farghaly A, Khalil Mahfouz AAR, El-Morshedy H, Farghaly

DOI: http://dx.doi.org/10.4314/ejhs.v29i1.14 
A, Khalil A. Ecological determinants of intestinal parasitic infections among pre-school children in an Urban Squatter Settlement of Egypt. J Trop Pediatr 1997; 43:341-44.

5. Erko B, Medhin G. Human helminthiasis in Wondo Genet, Southern Ethiopia, with emphasis on geohelminthiasis. Ethiop Med J 2003; 41:333.

6. Legesse M, Erko B. Prevalence of intestinal parasites among schoolchildren in a rural area close to the southeast of Lake Langano, Ethiopia. Ethiop J Health Dev 2004; 18:116.

7. M Amare, G Solomon, K Tesfaye. Prevalence of intestinal parasitic infections among urban dwellers in southwest Ethiopia. Ethiop J.Heal Dev 2007; 21(1):12-17.

8. De Silva NR, Brooker S, Hotez PJ, Montresor A, Engels D, Savioli L. Soil transmitted helminth infections: updating the global picture. Trends Parasitol 2003; 19:547-551.

9. Haileamlak Abraham. Intestinal parasites in asymptomatic children in Southwest Ethiopia. Ethiop J Health Sci 2005; 15 (2):107-118.

10. Limoncu M E, Kurt O, Gümüş M, Kayran E, Balcioğlu I C, Dinç G, Ozbilgin A. Is there an association between clinical symptoms and intestinal parasitic infections? Int $J$ Clin Pharmacol Res 2005; 25(3):151-154.

11. Rice J E, Skull S A, Pearce C, Mulholland N, Davie G, Carapetis J R. Screening for intestinal parasites in recently arrived children from East Africa. J Paediatr Child Health 2003; 39 (6):456.

12. Endris M, Lemma W, Belyhun Y, Moges B, Gelaw A, Angaw B. Prevalence of intestinal parasites and associated risk factors among students of Atse Fasil general elementary school, Azezo, Northwestern Ethiopia. Ethiop J Health Biomed Sci 2010; 3(1):25-33.

13. Asrat A, Tewodros D, Alemayehu W. Prevalence and risk factors of IPs among Delgi school children, Northern Gonder, Ethiopia. J Parasitol Vector Biol 2011; 3(5), 75-81.

14. Abate A, Kibret B, Bekalu E, Abera S, Teklu T, Yalew A et al. Cross-sectional study on the prevalence of intestinal parasites and associated risk factors in Teda health centre, Northwest Ethiopia. ISRN Parasitology 2013. http://dx.doi.org/10.5402/2013/757451.

15. Ibrahim M Muhammad, Askira M Umoru, Tom M Isyaka. Intestinal parasitic infections among patients attending a Tertiary Health Institution in Northeastern Nigeria. Am J Res Commun 2014; 2(6); 88-96.
16. HT Le, ID Brouwer, H Verhoef, KC Nguyen and FJ Kok. Anemia and intestinal parasite infection in school children in rural Vietnam. Asia Pac J Clin Nutr 2007; 16 (4):716-723.

17. Girum Tefera. Prevalence of intestinal parasitic infections among patients with diarrhea at Wonago health center, Southern Ethiopia: a retrospective study. Immunol Infect Dis 2015; 3(1): 1-6.

18. Girum T. The prevalence of intestinal helminthic infections and associated risk factors among school children in Babile town, eastern Ethiopia. Ethiop J Health Dev 2005; 19:140-147.

19. Tariq Masood Malik, Zahid Farooq Baig. Frequency and pattern of intestinal parasitic infestations in upper Neelum Valley. Pak Armed Forces Med J 2006; 4: 67-73.

20. Zaglool DAM, Khodari YAW, Gazzaz ZJ, Dhafar KO, Shaker HAS, Farook MV. Prevalence of intestinal parasites among patients of Al-Noor specialist Hospital, Makkah, Saudi Arabia. Oman Med J. 2011; 26(3):182-185.

21. Fekadu D, Beyene P, Amha K. Hookworm species distribution among school children in Asendabo Town, Jimma Zone, South west Ethiopia. Ethiop J Heal Sci 2008; 18:53-56.

22. Yami A, Mamo Y, Kebede S. Prevalence and predictors of intestinal helminthiasis among school childern in Jimma zone; a cross-sectional study. Ethiop J Heal Sci 2011; 21: 167-174.

23. Robert M Nyarango, Peninah A Aloo, Ephantus W Kabiru, Benson $\mathrm{O}$ Nyanchongi. The risk of pathogenic intestinal parasite infections in Kisii Municipality, Kenya. BMC Publ Heal 2008; 8.

24. Jamaiah I, Rohela M. Prevalence of Intestinal Parasites among Members of the public in Kuala Lumpur, Malaysia. Southeast Asian J Trop Med Pub Heal 2005; 36: 68-71.

25. H M El-Masry; Y. A. Ahmed; A. A. Hassan, et al. Prevalence, risk factors and impacts of schistosomal and intestinal parasitic infections among rural school children in Sohag Governorate. The Egyptian Journal of Hospital Medicine 2007; 29: 616-630.

26. Abossie A and Seid M. Assessment of the prevalence of intestinal parasitosis and associated risk factors among primary school children in Chencha town, Southern Ethiopia. BMC Publ Heal 2014; 14:166.

27. Cheesbrough M. District laboratory practice in tropical countries part $1,2^{\text {nd }}$ ed. New York. Cambridge University Press, 2009: 196-200.

DOI: http://dx.doi.org/10.4314/ejhs.v29i1.14 